\title{
CLUMPING AND THE INTERPRETATION OF kpc-SCALE MAPS OF THE INTERSTELLAR MEDIUM: SMOOTH H I AND CLUMPY, VARIABLE $\mathrm{H}_{2}$ SURFACE DENSITY
}

\author{
Adam K. Leroy ${ }^{1}$, Cheoljong LeE ${ }^{2}$, Andreas Schruba ${ }^{3}$, Alberto Bolatto ${ }^{4}$, Annie Hughes ${ }^{5}$, \\ Jérôme Petry ${ }^{6,7}$, Karin SANDSTrom $^{5}$, Eva Schinnerer $^{5}$, and Fabian Walter ${ }^{5}$ \\ ${ }^{1}$ National Radio Astronomy Observtory, 520 Edgemont Road, Charlottesville, VA 22903, USA \\ ${ }^{2}$ Department of Astronomy, University of Virginia, 530 McCormick Road, Charlottesville, VA 22904, USA \\ ${ }^{3}$ California Institute for Technology, 1200 E California Blvd, Pasadena, CA 91125, USA \\ ${ }_{5}^{4}$ Department of Astronomy, University of Maryland, College Park, MD 20742, USA \\ ${ }^{5}$ Max Planck Institute für Astronomie, Königstuhl 17, D-69117 Heidelberg, Germany \\ ${ }^{6}$ Institut de Radioastronomie Millimétrique, 300 Rue de la Piscine, F-38406 Saint Martin d'Héres, France \\ ${ }^{7}$ Observatoire de Paris, 61 Avenue de l'Observatoire, F-75014 Paris, France \\ Received 2013 February 14; accepted 2013 March 25; published 2013 May 7
}

\begin{abstract}
Many recent models consider the structure of individual interstellar medium (ISM) clouds as a way to explain observations of large parts of galaxies. To compare such models to observations, one must understand how to translate between surface densities observed averaging over large $(\sim \mathrm{kpc})$ scales and surface densities on the scale of individual clouds ( $\sim$ pc scale), which are treated by models. We define a "clumping factor" that captures this translation as the ratio of the mass-weighted surface density, which is often the quantity of physical interest, to the area-weighted surface density, which is observed. We use high spatial resolution (sub-kpc) maps of $\mathrm{CO}_{\text {and }} \mathrm{H}_{\mathrm{I}}$ emission from nearby galaxies to measure the clumping factor of both atomic and molecular gas. The molecular and atomic ISM exhibit dramatically different degrees of clumping. As a result, the ratio $\mathrm{H}_{2} / \mathrm{H}_{\mathrm{I}}$ measured at $\sim \mathrm{kpc}$ resolution cannot be trivially interpreted as a cloud-scale ratio of surface densities. H I emission appears very smooth, with a clumping factor of only $\sim 1.3$. Based on the scarce and heterogeneous high-resolution data available, $\mathrm{CO}$ emission is far more clumped with a widely variable clumping factor, median $\sim 7$ for our heterogeneous data. Our measurements do not provide evidence for a universal mass-weighted surface density of molecular gas, but also cannot conclusively rule out such a scenario. We suggest that a more sophisticated treatment of molecular ISM structure, one informed by high spatial resolution CO maps, is needed to link cloud-scale models to kpc-scale observations of galaxies.
\end{abstract}

Key words: galaxies: ISM - ISM: structure - stars: formation

\section{CLUMPING AND SURFACE DENSITIES IN ISM MAPS}

Observations of atomic and molecular gas now achieve spatial resolution of several hundred parsecs to a few kiloparsecs in large samples of nearby galaxies (e.g., Helfer et al. 2003; Walter et al. 2008; Leroy et al. 2009) or even small sets of high-redshift galaxies (Hodge et al. 2012; Tacconi et al. 2012). Such observations isolate key physical conditions such as stellar surface density, metallicity, or the interstellar radiation field. However, with a few exceptions, these observations still do not resolve individual clouds of atomic and molecular gas, which are often considered to be the fundamental units of the interstellar medium (ISM).

The interpretation of these observations often utilizes predictions from models that treat the surface density of gas on the scale of individual clouds. For example, the models of Krumholz et al. (2009a, 2009b), Wolfire et al. (2010), Feldmann et al. (2012), and Narayanan et al. (2012) all consider the structure of individual photodissociation regions or atomic-molecular complexes to explain observations on the scale of galaxies. In these models, the surface density of an individual cloud represents a key parameter, often because it indicates the degree of shielding from the ambient radiation field. Because these models focus on cloud structure, the mapping between the readily observed average, or "area-weighted," surface density at $\sim \mathrm{kpc}$ resolution and the cloud-scale, "mass-weighted," surface density represents an essential component of comparing observations and theory. This mapping is often referred to as "clumping" and quantified via a "clumping factor."
For the most part, the adopted clumping factors represent guesses informed by our coarse knowledge of ISM structure and giant molecular clouds (GMCs) but not directly based on observations. However, this factor can also be directly measured from high spatial resolution data. In this Letter we collect a large set of observations to measure the relationship between the surface density of the ISM averaged over large $(\sim \mathrm{kpc})$ scales and the "true" small-scale surface density. We consider both atomic $(\mathrm{HI})$ and molecular $\left(\mathrm{H}_{2}\right.$, traced by $\left.\mathrm{CO}\right)$ gas and discuss the implications of our calculation for the comparison to models.

We cast this discussion in terms of three quantities: the mass-weighted average surface density, $\langle\Sigma\rangle^{\mathrm{M}}$, the area-weighted average surface density, $\langle\Sigma\rangle^{\mathrm{A}}$, and a clumping factor, $c$, relating the two. The mass-weighted average surface density is

$$
\langle\Sigma\rangle^{\mathrm{M}}=\frac{\int_{A} \Sigma \times \Sigma d A}{\int_{A} \Sigma d A}=\frac{\int_{A} \Sigma^{2} d A}{\int_{A} \Sigma d A},
$$

where $\Sigma$ is the true gas mass surface density along a line of sight and the integral occurs over some area element $A$. Then the denominator is simply the sum of gas in that area, and the calculation returns the mass-weighted average surface density over the area. That is, $\langle\Sigma\rangle^{\mathrm{M}}$ is the column density at which most mass exists. Contrast this quantity with what is observed by a telescope for which a resolution element has size $A$,

$$
\langle\Sigma\rangle^{\mathrm{A}}=\frac{\int_{A} \Sigma d A}{\int_{A} d A} .
$$


That is, the telescope observes the area-weighted average surface density within the beam.

These two quantities, $\langle\Sigma\rangle^{\mathrm{M}}$ and $\langle\Sigma\rangle^{\mathrm{A}}$, will be the same for a smooth medium. They differ for a clumpy medium with most of the mass in small, high column density regions spread over large, low column density areas. In this case, $\langle\Sigma\rangle^{\mathrm{A}}$ may be much lower than $\langle\Sigma\rangle^{\mathrm{M}}$. We define a clumping factor, $c$, to quantify this distinction as

$$
c \equiv \frac{\langle\Sigma\rangle^{\mathrm{M}}}{\langle\Sigma\rangle^{\mathrm{A}}} .
$$

$c$ will be high for a clumpy, inhomogeneous medium and low for a smooth medium. It will never fall below unity. In practice, $\langle\Sigma\rangle^{\mathrm{M}}$ will be derived at finite resolution, so that $c$ could be more rigorously defined as $c_{a}^{b}$, the clumping factor calculated at final resolution $b$ with $\langle\Sigma\rangle^{\mathrm{M}}$ derived from data with intrinsic resolution $a$. In this Letter, $b$ will always be $1 \mathrm{kpc} ; a$ will vary from data set to data set.

The clumping factor, $c,\langle\Sigma\rangle^{\mathrm{A}}$, and $\langle\Sigma\rangle^{\mathrm{M}}$ give us a formalism to ask several questions related to the structure of the ISM and the interpretation of $\sim \mathrm{kpc}$ resolution elements.

1. How does the mass-weighted $\langle\Sigma\rangle^{\mathrm{M}}$ relate to the areaweighted, observable $\langle\Sigma\rangle^{\mathrm{A}}$ for kpc-resolution measurements of atomic and molecular gas in galaxies? What are typical clumping factors?

2. Is clumping the same for atomic and molecular gas, so that the $\mathrm{H}_{2}$-to-H I ratio at large scales may be readily interpreted in terms of cloud structure?

3. Can one reliably predict the surface density of individual regions-relevant to photon dominated region and cloud structure calculations-from coarse resolution measurements?

\section{DATA AND CALCULATIONS}

We assemble all readily available high spatial resolution $(\lesssim 500 \mathrm{pc}$ ) $\mathrm{CO}$ and $\mathrm{Hi}$ maps of nearby galaxies and use these to calculate $\langle\Sigma\rangle^{\mathrm{M}},\langle\Sigma\rangle^{\mathrm{A}}$, and $c$. We make use of three recent H I surveys of nearby galaxies: THINGS (Walter et al. 2008), LITTLE THINGS (Hunter et al. 2012), and VLA ANGST (Ott et al. 2012). We supplement these with a collection of $\mathrm{H}$ I data obtained to complement the HERACLES CO survey (presented in Leroy et al. 2012; Schruba et al. 2011; Sandstrom et al. 2012) and WSRT maps of M33 (Deul \& van der Hulst 1987) and M31 (Brinks \& Shane 1984). Whenever possible, we use the naturally weighted data. We include all galaxies from these surveys that have linear resolution better than $500 \mathrm{pc}$ and inclination less than $50^{\circ}$ (we except M31 and M33 from the inclination requirement). For the $\mathrm{H}_{\text {I }}$ calculation we consider only regions inside $r_{25}$ with column densities $N(\mathrm{H})>10^{20} \mathrm{~cm}^{-2}$. We use the integrated intensity maps provided by each survey in its data release.

High spatial resolution $\mathrm{CO}$ data remain harder to come by than high-resolution $\mathrm{H}$ I data because nearby dwarf galaxies tend to be faint in $\mathrm{CO}$ emission and the sensitivity of $\mathrm{mm}$ wave telescopes has been limited before ALMA. This scarcity leads us to assemble a heterogeneous collection of high-resolution CO. This includes the MAGMA (Wong et al. 2011) and NANTEN (Fukui et al. 1999) surveys of the LMC, the IRAM 30 m survey of CO in M31 (Nieten et al. 2006), the combined BIMA and FCRAO survey of M33 (Rosolowsky 2007), ALMA science verification data on the Antennae galaxies, and a handful of the brightest and nearest galaxies from BIMA SONG (NGC 2903, 3627, 5194, and 6946; Helfer et al. 2003). We supplement these with two new data sets: high-resolution CARMA mapping of select fields in M31 (PI: A. Schruba; A. Schruba et al., in preparation) and the Plateau de Bure Arcsecond Whirlpool Survey (PAWS; Schinnerer et al. 2013; Pety et al. 2013) of M51. Except for the ALMA Antennae data, all of these data sets target the $\mathrm{CO} J=1 \rightarrow 0$ line and include (sometimes exclusively) short-spacing data; the Antennae data target the $\mathrm{CO} J=3 \rightarrow 2$ and CO $J=2 \rightarrow 1$ transitions. ${ }^{8}$ Note that we have multiple data sets on several galaxies (M31, the LMC, and M51) and treat each data set, rather than galaxy, as a separate measurement. For comparison, we also calculate $c$ from the composite CO survey of the Milky Way by Dame et al. (2001), considering only intermediate latitude $\left(30^{\circ}>|b|>5^{\circ}\right)$ gas. We smooth their data with a 1.25 kernel to minimize sampling issues and integrate only over areas covered by the surveys.

Calculating moment zero maps. Because of the $\Sigma^{2}$ term in Equation (1), $\langle\Sigma\rangle^{\mathrm{M}}$ is not robust to the inclusion of noise in the calculation. We must therefore mask the data before carrying out our calculations. This is mostly an issue for the $\mathrm{CO}$ data, as the integrated intensity maps provided by the H I surveys have sufficient signal-to-noise ratio $(\mathrm{S} / \mathrm{N})$ for our purposes. For the $\mathrm{CO}$, we create new masks and re-derive integrated intensity maps for each data set. Typically, we estimate the noise from the empty regions of the cube, identify a core of high significance emission, often two successive channels with $\mathrm{S} / \mathrm{N}>5$, and expand this high significance core to include fainter but still significant emission. We integrate the masked data cube to produce a moment 0 map, which we use in further analysis. For the LMC maps from MAGMA and NANTEN and the M33 map, the $\mathrm{S} / \mathrm{N}$ at the native resolution is too low to yield a high-quality masked integrated intensity map. Therefore, we convolve the data to a slightly worse resolution before masking and any analysis.

This exercise produces maps well suited to derive $\langle\Sigma\rangle^{\mathrm{M}}$, but the process of masking at the native resolution does remove the possibility of picking up any contribution from a low S/ $\mathrm{N}$ diffuse component (e.g., Pety et al. 2013). Fundamentally, this is a limitation of the data themselves, and future, more sensitive surveys capable of detecting diffuse emission over individual lines of sight will improve this situation and quantify the contribution of faint, pervasive $\mathrm{CO}$ emission to the total molecular gas budget. ${ }^{9}$

When sampling the $\mathrm{CO}$ emission, we restrict ourselves to areas that include significant emission within the mask. Roughly, our criterion is that in the map smoothed to $1 \mathrm{kpc}$ resolution, the average brightness is such that we could have detected that line of sight at the original, higher resolution. That is, we consider areas where our sensitivity at high resolution is sufficient to detect the average brightness. This allows us to avoid "edge" or "clipping" effects in which only one small patch of bright emission is included in the beam, leading to high $\langle\Sigma\rangle^{\mathrm{M}}$ but low $\langle\Sigma\rangle^{\mathrm{A}}$. Because these "edges" are mostly present (within $r_{25}$ ) in the $\mathrm{CO}$ and not $\mathrm{H}_{\mathrm{I}}$ maps, including them would make our conclusions more extreme.

\footnotetext{
8 For purposes of calculating surface densities, we assume these lines to be thermalized. In actuality, they are likely somewhat sub-thermal but uncertainty is likely offset by a somewhat lower $\alpha_{\mathrm{CO}}$ in the Antennae. In any case, these conversion factors effectively divide out when calculating $c$.

9 This effect matters but does not appear to dominate our results. For example, if we add a pervasive CO component to the PAWS M51 data with magnitude $\sim 0.5$ times our sensitivity — an aggressive scenario-then $c$ drops from $\approx 6$ to $\approx 5.1$ over the region that we consider. Fainter regions, which we avoid, will be more affected.
} 


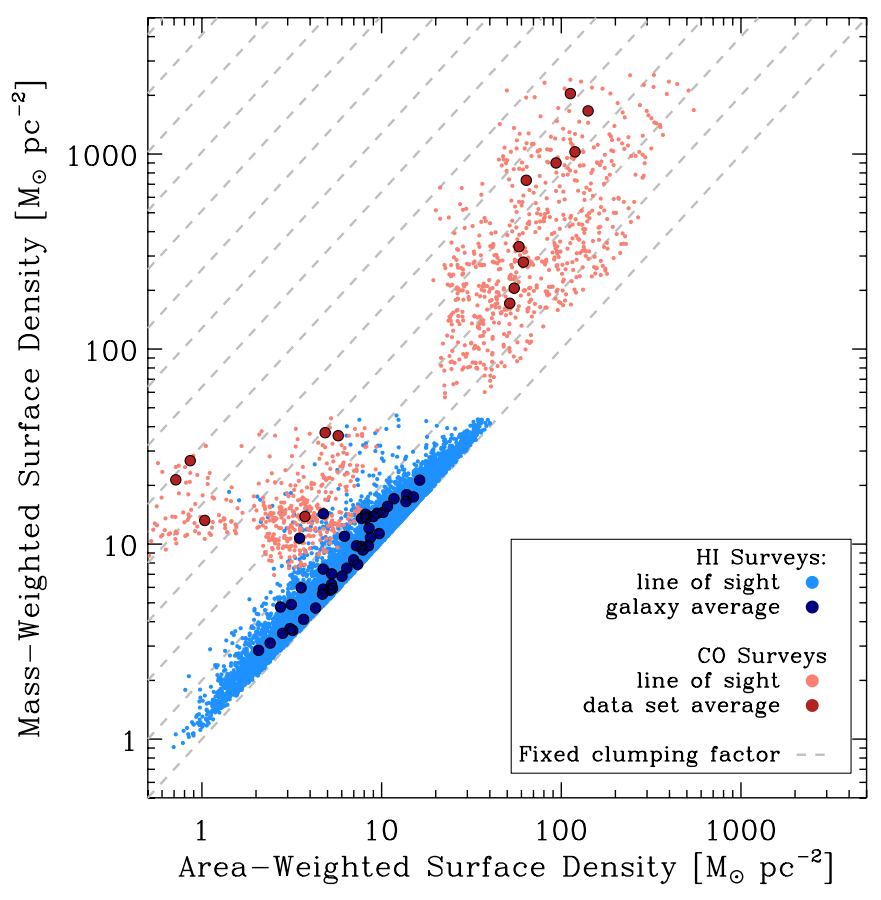

Figure 1. Mass-weighted surface density at $1 \mathrm{kpc}$ resolution ( $y$-axis), Equation (1), as a function of area-weighted surface density, Equation (2), at the same resolution ( $x$-axis) — note that no inclination corrections are applied, broadening the spread of apparent column densities. That is, the true surface density from which most emission arises as a function of the column density that would be measured at $1 \mathrm{kpc}$ resolution. Blue points show $\mathrm{H}$ I data and red points show $\mathrm{H}_{2}$ estimates from $\mathrm{CO}$ emission. Light points show individual lines of sight and dark points show averages for whole data sets. Gray lines show fixed clumping factors spaced by a factor of two. Hi shows good tracking between $\langle\Sigma\rangle^{\mathrm{M}}$ and $\langle\Sigma\rangle^{\mathrm{A}}$ with very low clumping factors, seldom above a factor of two. Conversely, $\mathrm{CO}$ exhibits a high degree of clumping, almost never less than a factor of two but often more than a factor of 10 and a scattered, non-universal relation between $\langle\Sigma\rangle^{\mathrm{M}}$ and $\langle\Sigma\rangle^{\mathrm{A}}$.

Deriving $\langle\Sigma\rangle^{\mathrm{M}},\langle\Sigma\rangle^{\mathrm{A}}$, and $c$. We assume that $21 \mathrm{~cm}$ and $\mathrm{CO}$ emission linearly trace the surface density of atomic and molecular gas, i.e., $\Sigma \propto I$, and calculate $\langle\Sigma\rangle^{\mathrm{M}}$ and $\langle\Sigma\rangle^{\mathrm{A}}$ following Equations (1) and (2). We convert from intensity to surface density adopting a fixed $\alpha_{\mathrm{CO}}=4.35 M_{\odot} \mathrm{pc}^{-2}$ $\left(\mathrm{K} \mathrm{km} \mathrm{s}^{-1}\right)^{-1}$ and $N(\mathrm{H} \mathrm{I})\left[\mathrm{cm}^{-2}\right]=1.823 \times 10^{18} I_{\mathrm{HI}}\left(\mathrm{K} \mathrm{km} \mathrm{s}^{-1}\right)$. Note that these factors divide out when calculating $c$.

To calculate $\langle\Sigma\rangle^{\mathrm{A}}$, we smooth from the native resolution to $1 \mathrm{kpc}$ using a normalized Gaussian kernel. To calculate $\langle\Sigma\rangle^{\mathrm{M}}$, we calculate $\Sigma^{2}$ at the native resolution, convolve this map to $1 \mathrm{kpc}$ resolution using a normalized Gaussian kernel, and then divide that map by $\langle\Sigma\rangle^{\mathrm{A}}$ following Equation (1). We record $\langle\Sigma\rangle^{\mathrm{M}}$, $\langle\Sigma\rangle^{\mathrm{A}}$, and the clumping factor, $c$, for a hexagonally spaced set of Nyquist-sampled (at $1 \mathrm{kpc}$ resolution) points.

After this exercise, we have $\approx 50,000$ data points from 46 galaxies for $\mathrm{HI}$ and $\approx 1000$ data points from 15 data sets in eight galaxies for $\mathrm{CO}$. As these numbers make clear, $\mathrm{CO}$ data represent the limiting reagent in this calculation, though thanks to ALMA, their prospect for short-term improvement is excellent.

\section{RESULTS}

Figures 1 and 2 and Table 1 report our results. Figure 1 shows $\langle\Sigma\rangle^{\mathrm{M}}$ as a function of $\langle\Sigma\rangle^{\mathrm{A}}$ for $\mathrm{CO}$ (red) and $\mathrm{H}_{\mathrm{I}}$ (blue) data. Figure 2 plots the clumping factor, $c$, as a function of the linear

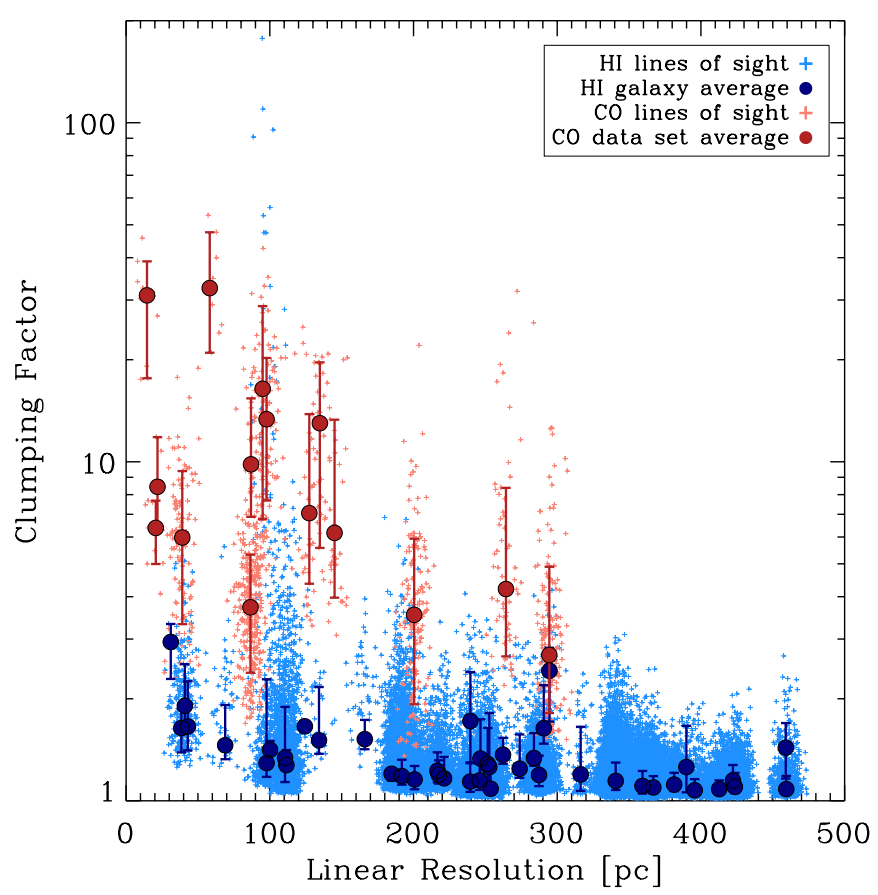

Figure 2. Clumping factor (y-axis), Equation (3), as a function of the linear resolution of the data used to derive the mass-weighted surface density, $\langle\Sigma\rangle^{\mathrm{M}}$, i.e., the native resolution of the data. The color scheme follows Figure 1. Again, $\mathrm{H}_{2}$ traced by $\mathrm{CO}$ appears much more strongly clumped than $\mathrm{H}$, exhibiting a wide range of clumping factors and tending to show a high degree of clumping. $\mathrm{H}$ I, by contrast, appears remarkably smooth, seldom exceeding clumping factors of two even at high spatial resolution. As in Figure 1, the difference in structure of the atomic and molecular medium and the variable clumpiness of the molecular medium are clearly evident.

Table 1

Clumping Factors for ISM Maps

\begin{tabular}{lcc}
\hline \hline Data Set & $\begin{array}{c}\text { Res. } \\
(\mathrm{pc})\end{array}$ & $\langle c\rangle$ \\
(1) & $(2)$ & $(3)$ \\
\hline & CO Data & \\
\hline M31 IRAM 30 m & 87 & $3.7_{-1.3}^{+1.6}$ \\
M31 CARMA "Brick 9" & 22 & $8.4_{-2.0}^{+3.4}$ \\
M31 CARMA "Brick 15" & 21 & $6.4_{-1.4}^{+1.3}$ \\
M33 BIMA+FCRAO & 98 & $13_{-5.7}^{+6.9}$ \\
LMC NANTEN & 58 & $33_{-12}^{+1.5}$ \\
LMC MAGMA & 15 & $31_{-13}^{+8.0}$ \\
M51 PAWS & 39 & $6.0_{-2.7}^{+3.4}$ \\
NGC 2903 BIMA SONG & 264 & $4.2_{-1.5}^{+4.2}$ \\
NGC 3627 BIMA SONG & 295 & $2.7_{-0.9}^{+2.2}$ \\
M51 BIMA SONG & 200 & $3.5_{-1.6}^{+2.4}$ \\
NGC 6946 BIMA SONG & 145 & $6.2_{-2.2}^{+7.1}$ \\
Antennae CO(2-1) North & 135 & $13_{-7.4}^{+6.7}$ \\
Antennae CO(2-1) South & 127 & $7.1_{-2.7}^{+6.8}$ \\
Antennae CO(3-2) North & 95 & $7.1_{-9.6}^{+12.4}$ \\
Antennae CO(3-2) South & 87 & $9.8_{-2.9}^{+5.6}$ \\
Local Milky Way (30 $>|b|>5^{\circ}$ ) & $\ldots$ & $\approx 6$ \\
\hline H I ensemble & & $1.26_{-0.15}^{+0.46}$ \\
$\ldots$ 0-250 pc resolution & & $1.34_{-0.17}^{+0.32}$ \\
$\ldots$ 250-500 pc resolution & & $1.19_{-0.11}^{+0.18}$ \\
\hline
\end{tabular}


resolution of the original data set used to calculate $\langle\Sigma\rangle^{\mathrm{M}}$. In both figures, light points show individual lines of sight and dark solid points show averages for whole data sets. Error bars on the whole galaxy points in Figure 2 show the $1 \sigma$ range for that data set. Table 1 reports the native resolution (after convolution to increase the $\mathrm{S} / \mathrm{N}$ in M33 and the LMC) and median clumping factor with $1 \sigma$ range for each $\mathrm{CO}$ data set. We report results for the ensemble of $\mathrm{H}_{\mathrm{I}}$ data, which Figures 1 and 2 demonstrate to be uniform.

These figures illustrate three points.

1. $\mathrm{H}_{\mathrm{I}}$ and $\mathrm{H}_{2}$ (traced by $\mathrm{CO}$ ) exhibit different clumping factors. Both Figures 1 and 2 show that essentially all of our $\mathrm{CO}$ data are more highly clumped than all of our H I data. The median clumping factor for a $\mathrm{CO}$ data set is $c=7$, while the median clumping factor for an $\mathrm{HI}$ data set is $c=1.26$. The specific cases of M33 and M31 illustrate this point cleanly. The M31 IRAM CO map shows clumping factor $\approx 4$; the M33 BIMA+FCRAO map shows clumping factor $\approx 13$. Both the M31 and M33 H I maps show clumping factor $\approx 1.3$.

As a direct result, a ratio of $\mathrm{H}_{2}$-to-H I surface densities obtained at large scales does not translate trivially into a ratio of surface densities at small scales. The assumption that the large-scale surface density in galaxies reflects the small-scale surface density in the same way for $\mathrm{H}_{2}$ and $\mathrm{H}$ I underlies the application of the Krumholz et al. (2009a) model to explain $\mathrm{H}_{2}$-to-H $\mathrm{I}$ ratios in galaxies. Though the physics of the model appear to apply successfully to individual clouds or regions (Bolatto et al. 2011; Lee et al. 2012), we suggest that more than a single "clumping" factor is necessary to make a rigorous comparison of the model to observations of large parts of galaxies.

Our calculation does not invalidate the kpc-resolution ratio of $\Sigma_{\mathrm{H}_{2}} / \Sigma_{\mathrm{H}_{\mathrm{I}}}$ as an interesting measurement. It simply suggests that this be viewed as a measure of mass balance among ISM phases over a large area and not indicative of small-scale ISM structure.

2. HI is very smooth. This conclusion leaps out of both figures. Even with high linear resolution, H i column density maps remain smooth and only weakly clumped. This can be explained by most $21 \mathrm{~cm}$ emission originating not from clumped bound clouds, but a diffuse medium with a high volume filling factor. The $\mathrm{H}$ i clumping factor does depend weakly on scale, a reasonable functional form is $c=392\left(l_{\mathrm{pc}}+100\right)^{-1.27}+1$, where $l_{\mathrm{pc}}$ is the (FWHM) linear resolution in parsecs.

3. $C O$ is clumpy with a wide range of $c$, making it hard to predict $\langle\Sigma\rangle^{\mathrm{M}}$ from $\langle\Sigma\rangle^{\mathrm{A}}$. In contrast to $\mathrm{H} \mathrm{I}, \mathrm{H}_{2}$ traced by $\mathrm{CO}$ emission appears clumpy with a wide range of $c$. The median among all data sets is $\approx 7$ with a factor of $2-3 \mathrm{rms}$ scatter among measurements. This is also close to the value that we estimate for the solar neighborhood from intermediate latitude gas, but we caution that we expect $c$ to change with improving native resolution of the data. Because we consider only bright regions, this represents a conservative estimate; the edges and faint regions that we exclude tend to have high $c$. The molecule-poor systems (M33 and the LMC) in the sample show the highest $c$, perhaps because they contain more isolated clouds and perhaps because their somewhat low metallicities lead any diffuse $\mathrm{H}_{2}$ component to emit less in $\mathrm{CO}$ (below some metallicity the clumping of $\mathrm{CO}$ emission and $\Sigma_{\mathrm{H}_{2}}$ will dramatically diverge).

We also note two less secure points implied by the data but requiring more aggressive assumptions about the $\mathrm{CO}-$ to- $\mathrm{H}_{2}$ conversion factor.

4. The total $\left(\mathrm{H}_{2}+\mathrm{HI}_{\mathrm{I}}\right)$ clumping factor must vary significantly among and within galaxies. We can only calculate the clumping factor for the total $\left(\mathrm{H}_{2}+\mathrm{HI}\right)$ gas in M31, M33, and the LMC. In each case the median $c$ is low $(\sim 1.3, \sim 1.3$, and $\sim 1.5)$, resembling that of the $\mathrm{H}_{\mathrm{I}}$. This is not surprising because for fixed $\alpha_{\mathrm{CO}}$, the $\mathrm{H}$ I mass exceeds the $\mathrm{H}_{2}$ mass in these galaxies by more than an order of magnitude, with $\mathrm{H}_{2}$ making up most of the gas along only a small fraction of the lines of sight at our resolution (M31 is more moleculerich than the other two, but still H I dominated). Generally, we expect that across most of the area in dwarf galaxies, which tend to be low metallicity and $\mathrm{HI}$-dominated, $c$ will resemble the $\sim 1.3$ that we measure for $\mathrm{HI}$. The outer parts of most spirals also tend to be $\mathrm{H}_{\mathrm{I}}$ dominated and should show similar values, while in the molecule-rich central parts of actively star-forming galaxies the values will more closely resemble the higher $c$ that we find for M51, NGC 2903, NGC 3627, and NGC 6946.

5. CO exhibits a wide range of $\langle\Sigma\rangle^{\mathrm{M}}$. In contrast to the common assumption that $\mathrm{CO}$ emerges from a population of fixed surface density clouds, the CO data in Figure 1 span two orders of magnitude in $\langle\Sigma\rangle^{\mathrm{M}}$. The figure does not provide good evidence that $\mathrm{CO}$ emerges from a fixed $\langle\Sigma\rangle^{\mathrm{M}}$ at high spatial resolution. In fact, the highest resolution data sets span roughly an order of magnitude (for fixed $\alpha_{\mathrm{CO}}$ ) in $\langle\Sigma\rangle^{\mathrm{M}}$ from the LMC $\left(\sim 25 M_{\odot} \mathrm{pc}^{-2}\right)$ to M51 $\left(\sim 330 M_{\odot} \mathrm{pc}^{-2}\right)$. The spread in $\langle\Sigma\rangle^{\mathrm{M}}$ may arise from sources other than the cloud scale surface density: variations in inclination and the conversion factor, superposition of fixed surface density clouds, the convolution of bound clouds with a diffuse background, or a lack of spatial resolution matched to individual clouds. However, our best guess is that the large range in apparent $\langle\Sigma\rangle^{\mathrm{M}}$ visible in Figure 1 in fact reflects a systematic dependence of cloud surface density on environment. This reinforces the thorough analysis of Hughes et al. (2013), who compare CO maps of M51 (PAWS), the LMC (MAGMA), and M33 and conclusively demonstrate fundamental differences between the volume and surface density probability density functions among and within the three galaxies (see also the spread in Milky Way $\Sigma$ discussed by Bolatto et al. 2013).

Our calculations show that the structures of the molecular and atomic ISM are more complex than have been assumed while vetting recent models. We suggest the "clumping factor" approach defined in Section 1 to quantify this structure and aid interpretation of lower resolution observations. With ALMA, we now able to easily obtain high-resolution, high-sensitivity ISM maps, so we expect such calculations to be feasible in many systems over the coming years.

We thank the referee for a constructive report and Scott Schnee and Mark Krumholz for feedback on drafts. We acknowledge the BIMA SONG, LITTLE THINGS, and VLA ANGST collaborations for making their data public. We thank IRAM for making the moment 0 map of M31 public. This Letter makes use of the following ALMA data: 
ADS/JAO.ALMA\#2011.0.00003.SV. ALMA is a partnership of ESO (representing its member states), NSF (USA), and NINS (Japan), together with NRC (Canada) and NSC and ASIAA (Taiwan), in cooperation with the Republic of Chile. The Joint ALMA Observatory is operated by ESO, AUI/NRAO, and NAOJ. The National Radio Astronomy Observatory is a facility of the National Science Foundation operated under cooperative agreement by Associated Universities, Inc. A.B. acknowledges partial support from grants NSF AST-0838178, NSF AST-0955836, and a Cottrell Scholar award from the Research Corporation for Science Advancement. K.S. is supported by a Marie Curie International Incoming fellowship. A.H. acknowledges funding from the Deutsche Forschungsgemeinschaft via grants SCHI 536/5-1 and SCHI 536/7-1 as part of the priority program SPP 1573 "ISM-SPP: Physics of the Interstellar Medium." J.P. was partially funded by the grant ANR-09-BLAN-0231-01 from the French Agence Nationale de la Recherche as part of the SCHISM project.

\section{REFERENCES}

Bolatto, A. D., Leroy, A. K., Jameson, K., et al. 2011, ApJ, 741, 12 Bolatto, A. D., Wolfire, M., \& Leroy, A. K. 2013, arXiv:1301.3498 Brinks, E., \& Shane, W. W. 1984, A\&AS, 55, 179

Dame, T. M., Hartmann, D., \& Thaddeus, P. 2001, ApJ, 547, 792
Deul, E. R., \& van der Hulst, J. M. 1987, A\&AS, 67, 509

Feldmann, R., Gnedin, N. Y., \& Kravtsov, A. V. 2012, ApJ, 747, 124

Fukui, Y., Mizuno, N., Yamaguchi, R., et al. 1999, PASJ, 51, 745

Helfer, T. T., Thornley, M. D., Regan, M. W., et al. 2003, ApJS, 145, 259

Hodge, J. A., Carilli, C. L., Walter, F., et al. 2012, ApJ, 760, 11

Hughes, A., Meidt, S. E., Schinnerer, E., et al. 2013, ApJ, in press (arXiv:1304.1219)

Hunter, D. A., Ficut-Vicas, D., Ashley, T., et al. 2012, AJ, 144, 134

Krumholz, M. R., McKee, C. F., \& Tumlinson, J. 2009a, ApJ, 693, 216

Krumholz, M. R., McKee, C. F., \& Tumlinson, J. 2009b, ApJ, 693, 216

Lee, M.-Y., Stanimirović, S., Douglas, K. A., et al. 2012, ApJ, 748, 75

Leroy, A. K., Bigiel, F., de Blok, W. J. G., et al. 2012, AJ, 144, 3

Leroy, A. K., Bolatto, A., Bot, C., et al. 2009, ApJ, 702, 352

Narayanan, D., Krumholz, M. R., Ostriker, E. C., \& Hernquist, L. 2012, MNRAS, 421, 3127

Nieten, C., Neininger, N., Guélin, M., et al. 2006, A\&A, 453, 459

Ott, J., Stilp, A. M., Warren, S. R., et al. 2012, AJ, 144, 123

Pety, J., Schinnerer, E., Leroy, A. K., et al. 2013, ApJ, in press (arXiv: 1304.1396)

Rosolowsky, E. 2007, ApJ, 654, 240

Sandstrom, K. M., Leroy, A. K., Walter, F., et al. 2012, arXiv:1212.1208

Schinnerer, E., Meidt, S. E., Pety, J., et al. 2013, ApJ, in press (arXiv:1304.1801)

Schruba, A., Leroy, A. K., Walter, F., et al. 2011, AJ, 142, 37

Tacconi, L. J., Neri, R., Genzel, R., et al. 2012, arXiv:1211.5743

Walter, F., Brinks, E., de Blok, W. J. G., et al. 2008, AJ, 136, 2563

Wolfire, M. G., Hollenbach, D., \& McKee, C. F. 2010, ApJ, 716,1191

Wong, T., Hughes, A., Ott, J., et al. 2011, ApJS, 197, 16 